\title{
Efficacy and Safety of Chemoradiation Therapy Using One-Shot Cisplatin via Hepatic Arterial Infusion for Advanced Hepatocellular Carcinoma with Major Macrovascular Invasion: a Single-arm Retrospective Cohort Study
}

\section{Kensuke Naruto}

Department of Gastroenterology and Metabolism, Hiroshima University

\section{Tomokazu Kawaoka}

Department of Gastroenterology and Metabolism, Hiroshima University

\section{Kenichiro Kodama}

Department of Gastroenterology and Metabolism, Hiroshima University

\section{Yutaro Ogawa}

Department of Gastroenterology and Metabolism, Hiroshima University

Kei Amioka

Department of Gastroenterology and Metabolism, Hiroshima University Yuki Yoshikawa

Department of Gastroenterology and Metabolism, Hiroshima University Chihiro Kikukawa

Department of Gastroenterology and Metabolism, Hiroshima University

\section{Yousuke Suehiro}

Department of Gastroenterology and Metabolism, Hiroshima University

\section{Kenji Yamaoka}

Department of Gastroenterology and Metabolism, Hiroshima University

\section{Yuwa Ando}

Department of Gastroenterology and Metabolism, Hiroshima University

\section{Yumi Kosaka}

Department of Gastroenterology and Metabolism, Hiroshima University

\section{Shinsuke Uchikawa}

Department of Gastroenterology and Metabolism, Hiroshima University

\section{Takashi Nakahara}

Department of Gastroenterology and Metabolism, Hiroshima University

\section{Eisuke Murakami}

Department of Gastroenterology and Metabolism, Hiroshima University 


\section{Atsushi Ohno}

Department of Gastroenterology and Metabolism, Hiroshima University

\section{Takuro Uchida}

Department of Gastroenterology and Metabolism, Hiroshima University

\section{Masami Yamauchi}

Department of Gastroenterology and Metabolism, Hiroshima University

\section{Wataru Okamoto}

Department of Gastroenterology and Metabolism, Hiroshima University

\section{Shoichi Takahashi}

Department of Gastroenterology and Metabolism, Hiroshima University

\section{Michio Imamura}

Department of Gastroenterology and Metabolism, Hiroshima University

\section{Keigo Chosa}

Diagnostic Radiology, Hiroshima University

\section{Kazuo Awai}

Diagnostic Radiology, Hiroshima University

\section{Katsumaro Kubo}

Therapeutic Radiology, Institute and Graduate School of Biomedical Sciences Hiroshima University

\section{Yasushi Nagata}

Therapeutic Radiology, Institute and Graduate School of Biomedical Sciences Hiroshima University

\section{Kazuaki Chayama}

Research Center for Hepatology and Gastroenterology, Hiroshima University

Hiroshi Aikata ( $\square$ aikata@hiroshima-u.ac.jp)

Department of Gastroenterology and Metabolism, Hiroshima University

\section{Research Article}

Keywords: hepatocellular carcinoma, macrovascular invasion, hepatic arterial infusion chemotherapy, radiation therapy

Posted Date: December 28th, 2021

DOI: https://doi.org/10.21203/rs.3.rs-1144509/v1

License: (c) (i) This work is licensed under a Creative Commons Attribution 4.0 International License. Read Full License 


\section{Abstract}

Background: Patients with hepatocellular carcinoma (HCC) and macrovascular invasion (MVI) who receive systemic chemotherapy have a poor prognosis. This study aimed to determine if one-shot cisplatin (CDDP) chemotherapy via hepatic arterial infusion (HAI) combined with radiation therapy (RT) prior to systemic chemotherapy could improve the outcomes of these patients.

Methods: This study consisted of $32 \mathrm{HCC}$ patients with the following eligibility criteria: (i) portal vein invasion 3/4 and/or hepatic vein invasion 2/3; (ii) received one-shot CDDP via HAl; (iii) received RT for MVI, (iv) a Child-Pugh score $\leq 7$; and (v) an Eastern Clinical Oncology Group Performance Status score of 0 or 1 . To determine the therapeutic effect, we collect the information of patients' characteristics and took contrast-enhanced computed tomography at the start of the therapy and every 2 to 4 months after the therapy initiated. We evaluated the overall response of the tumor and tumor thrombosis according to modified Response Evaluation Criteria in Solid Tumors. We assessed the patient's data statistically by the Mann-Whitney U-test, fisher's exact test, and evaluated overall survival and progress-free survival by logranked test, and analyzed the predictive factors by as appropriate.

Results: The overall response rate at the first evaluation performed a median of 1.4 weeks after HAl was $16 \%$ of the main intrahepatic tumor and $59 \%$ of the MVI. The best responses were the same as those of the first-time responses. The duration of median survival was 8.6 months, and for progression-free survival of the main intrahepatic tumor was 3.2 months. The predictive factor of overall survival was the relative tumor volume in the liver and the first therapeutic response of MVI. There were no severe adverse events or radiation-induced hepatic complications.

Conclusions: One-shot CDDP via HAI and RT were well tolerated and showed immediate and favorable control of MVI. Thus, this combination shows potential as a bridging therapy to systemic chemotherapy.

\section{Introduction}

In global cancer statics, Hepatocellular carcinoma (HCC) is a large abandon of cancer-related death [1]. Furthermore, the prognosis of HCC patients with macrovascular invasion (MVI) is poor [2]. HCC frequently occurs in patients with chronic hepatitis or liver cirrhosis due to hepatitis $\mathrm{B}$ or $\mathrm{C}$ viral infection, alcohol use, nonalcoholic steatohepatitis, or diabetes [3]. The survival of patients with HCC has improved with the development of diagnostic modalities and progress in treatment options, such as radical surgical resection, radiofrequency ablation, microwave ablation, percutaneous ethanol injection [4,5], and transcatheter arterial chemoembolization [6,7], radiation therapy (RT) [8], chemotherapy via hepatic arterial infusion (HAl), and multiple molecular targeting agents including immune checkpoint inhibitor $[9,10]$. However, the prognosis of advanced HCC patients, especially those with advanced MVI, remains poor [11-21].

Systemic chemotherapy such as the combination of atezolizumab plus bevacizumab (atezo+beva) is effective for patients with advanced HCC $[10,22,23]$ and for patients with HCC with portal vein invasion 
$(\mathrm{Vp})$, but the overall survival (OS) of the patients with $\mathrm{Vp}$ was less than that of patients without $\mathrm{Vp}$ [2325]. The poor prognosis of the patients with $\mathrm{Vp}$ is a result of major MVI that causes deterioration in the preserved hepatic function and portal hypertension, and interferes with the administration of chemotherapy [10]. However, atezo+beva take 2.8 months to obtain a response, and $20 \%$ of the recipients of these agents are nonresponders [23-25].

Thus, we aimed for a better outcome of patients with advanced HCC with the use of one-shot cisplatin (CDDP) via HAI plus RT to obtain the rapid control of MVI before the administration of systemic chemotherapy. HAl, used for either continual infusion via an implanted reservoir system or to administer one-shot CDDP, remains insufficient by itself to control MVI. Therefore, we attempted to examine the effects of combining one-shot CDDP via HAI with RT for patients with MVI.

Previously we reported on a study of the effects of treating patients with HCC by combining CDDP administered via an implanted reservoir system with RT [26-31]; however, there were complications with the implanted reservoir system. Moreover, only a few studies are available on one-shot CDDP via HAI combined with RT. Therefore, this study aimed to evaluate the safety and efficacy of one-shot CDDP via HAl combined with RT for patients before systemic chemotherapy.

\section{Materials And Methods \\ 2.1 Patients}

In this single-arm retrospective cohort study, patients with HCC were treated with one-shot CDDP via HAI plus RT for severe MVI. Severe MVI contains the tumor thrombus in the hemilobar branch of the portal vein (Vp3), the tumor thrombus in the main trunk or bilobar branches of the portal vein (Vp4), tumor thrombus in the main trunk of left hepatic vein, middle hepatic vein, or right hepatic vein (Vv2), and the tumor thrombus in inferior vena cava (Vv3).

The patients who met the following criteria were enrolled: (i) presence of Vp3-4 or Vv2-3, (ii) Child-Pugh score of 5-7, (iii) Eastern Clinical Oncology Group Performance Status score of 0-1, (iv) no history of systemic therapy, and (v) HAI performed from September 2004 to September 2020. This study was approved by the Institutional Review Board of Hiroshima University Hospital, and was based on the ethical principles of the Declaration of Helsinki 7th version on 2013 (project identification code number: E968). Detailed written informed consent was obtained from each patient for each treatment.

\subsection{Therapeutic Protocol}

\subsubsection{HAl}

In Japan, the chemotherapy via HAl with single bolus injection of CDDP (one-shot CDDP) is common treatment option for HCC patients with MVI. The effectiveness of one-shot HAl was previously reported [30]. The patients were administered CDDP at a dose of $65 \mathrm{mg} / \mathrm{m}^{2}$ (maximum dose, $100 \mathrm{mg} / \mathrm{m}^{2}$ ) at a rate 
of $2 \mathrm{~mL} / \mathrm{min}$ through a percutaneous catheter into the entire liver. The dose was decreased by $75 \%$ if the patient's estimated glomerular filtration rate was $<60 \mathrm{mg} / \mathrm{min} / 1.73 \mathrm{~m}^{2}$. When CDDP was infused into a part of the liver, the dose was adjusted roughly by the proportion of the tumor volume in the liver, which was determined on computed tomography (CT) images. Adequate hydration was also ensured both before and after the administration of CDDP to prevent CDDP-induced renal dysfunction.

\subsubsection{RT for MVI}

Every study patient was administered three-dimensional conformal radiation therapy (3D-CRT) concomitantly with one-shot CDDP via HAl. The treatments were administered in the Division of Radiation Oncology at our hospital [19]. The treatment protocol was as follows: 3D-CRT was performed with 18,10 , or $6 \mathrm{MV}$ high-energy photon beams that were delivered by a linear accelerator (CLINAC 2300 C/D or CLINAC iX linear accelerator; Varian Medical Systems Inc., Palo Alto, CA, USA) using the 3D conformal technique. Planning CT was used to determine the total volume of tumor involved in the MVI. The clinical target volume was established as the gross tumor volume plus the intrahepatic tumor volume in the basal portion of the MVI. The planning target volume comprised the clinical target volume plus a 10-20-mm margin in all directions to account for internal motion and set-up errors. Data consisting of the outlined target volume; total volume of liver tissue; and volume of at-risk structures, including the spinal cord, both kidneys, and nearby targets of the intestinal tract; were transferred to the treatment planning system (Pinnacle 3; Philips Medical Systems, Eindhoven, the Netherlands) with reference to the diagnostic contrast-enhanced CT images. The irradiation dose was 39 Gy depending on the volume of normal tissue and liver function, with $95 \%$ of the planning target volume receiving at least $95 \%$ of the irradiation dose.

\subsection{Assessment of Treatment Efficacy}

The therapeutic effectiveness was evaluated according to changes in the volumes of the tumors. The response to treatment was assessed by contrast-enhanced $\mathrm{CT}$. The base line CT image was taken before HAI. The image for the first-time evaluation was taken after the RT for MVI finished, and then every 2-3 months for follow-up. Especially, regarding a hypervascular tumor such as HCC, a decrease in the contrasted tumor volume during the arterial phase, as determined by contrast-enhanced CT, often indicates a decrease in tumor activity prior to tumor necrosis. Thus, response was determined according to the modified Response Evaluation Criteria in Solid Tumors (mRECIST) [32]. According to mRECIST, the therapeutic effect of the treatment on MVI was determined by measuring the longest diameter and the degree of contrast of the MVI.

\subsection{Evaluation of Treatment-Related Adverse Events}

The safety assessment included the documentation of adverse drug reactions every physical examination, the measurements of vital signs, and the results of clinical laboratory data. Adverse drug reactions were defined according to the National Cancer Institute Common Terminology Criteria for Adverse Events (NCl-CTCAE) v5.0 (http://ctep.cancer.gov/protocolDevelopment/electronic_applications/docs/ctcaev3.pdf). 
Radiation-induced liver disease (RILD) was divided into "classical" and "non-classical" RILD [33]. The endpoint of non-classical RILD was described in patients with poor liver function. Classical RILD, which usually occurs between 2 weeks and 3 months after irradiation, involves anicteric hepatomegaly and ascites, and elevation of the alkaline phosphatase level to at least 2-fold the upper limit of normal or the pretreatment value in the absence of tumor progression. Classical RILD can occur in patients with good liver function. Non-classical RILD, which usually occurs between 1 week and 3 months after irradiation, involves elevation of the alkaline phosphatase level to greater than 5-fold the upper limit of normal. Furthermore, it involves CTCAE grade 4 in patients with a baseline value greater than 5-fold the upper limit of normal within 3 months after the completion of RT, or a decrease in liver function (defined by an increase in the Child-Pugh score of $>2$ points), in the absence of classical RILD.

\subsection{Statistical Analysis}

The Mann-Whitney U-test and the Fisher exact test were used for statistical analysis of patients' characteristics, as appropriate. We estimated the overall survival (OS) and progression-free survival (PFS). The OS was calculated from the initial date of therapy. PFS was calculated with the dates of initial therapy and the diagnosis of progressive disease (PD). These parameters were plotted and assessed by the Kaplan-Meier life-table method, and differences between survivals of subgroups were evaluated by the log-rank test. Multivariate analysis of predictors of OS was assessed by binomial logistic regression with backward elimination according to the $p$ value. $P<0.10$ was considered to demonstrate a tendency, whereas $p<0.05$ was considered to be statistically significant. EZR (Saitama Medical Center, Jichi Medical University, Saitama, Japan), which is a graphical user interface for R (The R Foundation for Statistical Computing, Vienna, Austria) was used for statistical analysis. EZR is a modified version of R commander that was created to add statistical functions that are frequently used in biostatistics [34].

\section{Results}

\subsection{Patient Clinical Characteristics at the Start of HAI}

A total of 32 patients were enrolled in this retrospective cohort study. The characteristics of the patients and clinical data at the time of initial treatment are summarized in Table 1 . The median age of the patients was 69.5 years (range, 40-85 years), and 28 patients were men. Child-Pugh scores of 5, 6, and 7 were noted in 9 (28\%), 10 (31\%), and 13 (41\%) patients, respectively. Regarding the etiology of HCC, 9 and 10 patients were positive for hepatitis B surface antigen and hepatitis $\mathrm{C}$ virus antibodies, respectively; and 1 patient was positive for both viruses. The relative tumor volume in the liver was < 50\% in 19 (59\%) patients. The median size of the liver tumors was $103 \mathrm{~mm}$ (range, 36-185 mm). The number of patients with HCC with Vp4 was 15 (47\%), and those with Vp3 was 13 (41\%). A total of 10 patients (31\%) had extrahepatic metastases. The median alpha-fetoprotein (AFP) level was $713.2 \mathrm{ng} / \mathrm{mL}$ (range, 1.3$3,686,000 \mathrm{ng} / \mathrm{mL}$ ). The median des-y-carboxy prothrombin level was $10,039 \mathrm{mAU} / \mathrm{mL}$ (range, 36-327,600 $\mathrm{ng} / \mathrm{mL}$ ). A total of 23 patients received additional systemic therapy; 6 received sorafenib, and 13 received 
lenvatinib, and 4 received investigational agents such as pembrolizumab and the combination of durvalumab and tremelimumab. 
Table 1

Clinical characteristics of patients who received one-shot CDDP via HAI plus RT

\section{Characteristics}

Age (years)

Gender (male/female)

ECOG-Performance Status (0/1)

Etiology (HBV/HCV/HBV+HCV/other)

Total bilirubin $(\mathrm{mg} / \mathrm{dL})$

Albumin $(\mathrm{g} / \mathrm{dL})$

Prothrombin consumption test (\%)

Child-Pugh score $(5 / 6 / 7)$

ALBI score

mALBI grade $(1 / 2 \mathrm{a} / 2 \mathrm{~b} / 3)$

Size of liver tumor ( $\mathrm{mm}$ )

Number of intrahepatic tumors $(<4 / \geq 4)$

Relative tumor volume in the liver

$(<50 \% / \geq 50 \%)$

$\mathrm{Vp}(3 / 4)$

$\operatorname{Vv}(0 / 1 / 2 / 3)$

Extrahepatic spread (without/with)

HCC stage (III/IVa/IVb) ${ }^{\mathrm{a}}$

$\mathrm{BCLC}$ stage $(\mathrm{A} / \mathrm{B} / \mathrm{C})$

Alpha-fetoprotein $(\mathrm{ng} / \mathrm{mL})$

Des-y-carboxy prothrombin $(\mathrm{mAU} / \mathrm{mL})$

Additional systemic therapy (with/without)

\section{Median (range) or number of patients}

$28 / 4$

$25 / 7$

$9 / 10 / 1 / 12$

$0.9(0.4-1.5)$

$3.5(2.6-4.6)$

$86.0(6.2-111)$

$9 / 10 / 13$

$-2.17(-3.24--1.28)$

$5 / 7 / 19 / 1$

$103.2(36-185)$

$18 / 14$

$19 / 13$

$13 / 15$

$27 / 1 / 1 / 3$

$22 / 10$

$4 / 6 / 22$

$0 / 0 / 32$

$713.2(1.3-3,686,000)$

$10,039(36-327,600)$

$23 / 9$

The clinical characteristics of the HCC patients with MVI who received one-shot CDDP chemotherapy via HAl plus RT. Abbreviations: HCC, hepatocellular carcinoma; MVI, macrovascular invasion; HAl, hepatic arterial infusion; CDDP, cisplatin; HAl, hepatic arterial infusion; RT, radiation therapy; ECOGPerformance Status, Eastern Cooperative Oncology Group Performance Status; HBV, hepatitis B virus; $\mathrm{HCC}$, hepatocellular carcinoma; HCV, hepatitis C virus; ALBI score $=\log _{10}$ ([total-

bilirubin $(\mathrm{mg} / \mathrm{dL})]^{\star} 17.1 * 0.66$-[albumin $\left.(\mathrm{g} / \mathrm{dL})\right]^{\star} 10 * 0.085$; mALBI grade $1: 2 \mathrm{a}: 2 \mathrm{~b}: 3=$ ALBI score $\leq-2.6$ : $>-2.6$ to <-2.27: $\geq-2.27$ to $\leq-1.39$ : $>-1.39$; Vp, portal vein invasion; $\mathrm{Vv}$, venous invasion; BCLC stage, Barcelona Clinic liver cancer stage. ${ }^{a}$ According to the Liver Cancer Study Group of Japan. 


\subsection{Overall Response Rate to HAl and RT}

Based on the mRECIST, which evaluates the changes in volume as determined by contrast-enhanced CT, the best overall response rate (ORR) was $16 \%$. The best ORRs of tumor involved in $\mathrm{MVI}$ and the main tumor were $59 \%$ and $16 \%$, respectively. The disease control rates (DCR) of tumor involved in MVI and the main tumor were $97 \%$ and $41 \%$, respectively. The complete response (CR), partial response (PR), stable disease (SD), and progressive disease (PD) rates of the first-time response assessment of the MVI were $0 \%, 59 \%, 28 \%$, and $3 \%$, respectively; and those of the main tumor were $0 \%, 16 \%, 25 \%$, and $59 \%$, respectively (Table 2 ).

Table 2

Responses of tumor in MVI and main tumor to one-shot CDDP chemotherapy plus RT.

\begin{tabular}{|c|c|c|c|c|c|c|}
\hline & \multicolumn{6}{|c|}{ HAl+RT $(n=32)$} \\
\hline & \multirow{2}{*}{\multicolumn{3}{|c|}{$\begin{array}{l}\text { First-time response } \\
\text { (median, } 1.4 \text { month) }\end{array}$}} & \multirow{2}{*}{\multicolumn{3}{|c|}{ Best response }} \\
\hline & & & & & & \\
\hline & Whole tumor & Main tumor & $\mathrm{MVI}$ & Whole tumor & Main tumor & $\mathrm{MVI}$ \\
\hline $\mathrm{CR}$ & 0 & 0 & 0 & 0 & 0 & 0 \\
\hline PR & $16(5)$ & $16(5)$ & 59 (19) & $16(5)$ & $16(5)$ & 59 (19) \\
\hline SD & $25(8)$ & $25(8)$ & $28(12)$ & $25(8)$ & $25(8)$ & $28(12)$ \\
\hline PD & $59(19)$ & $59(19)$ & $3(1)$ & $59(19)$ & $59(19)$ & $3(1)$ \\
\hline ORR & $16(5)$ & $16(5)$ & 59 (19) & $16(5)$ & $16(5)$ & 59 (19) \\
\hline DCR & $41(13)$ & $41(13)$ & $97(31)$ & $41(13)$ & $41(13)$ & $97(31)$ \\
\hline \multicolumn{7}{|c|}{$\begin{array}{l}\text { The best and first-time response of tumor in MVI and main tumor to one shot CDDP chemotherapy via } \\
\text { HAI plus RT, as assessed by mRECIST. The median period of the first-time response was assessed } \\
\text { was } 1.4 \text { months from HAI. Data are presented as percentages (numbers). Abbreviations: MVI, } \\
\text { macrovascular invasion; CDDP, cisplatin; RT, radiation therapy; CR, complete response; PR, partial } \\
\text { response; SD, stable disease; PD, progressive disease; ORR, overall response rate; DCR, disease contro } \\
\text { rate; mRECIST, modified Response Evaluation Criteria in Solid Tumors. }\end{array}$} \\
\hline
\end{tabular}

\subsection{OS and Factors Affecting OS}

The median OS time of the study patients was 8.6 months (Figure 1A). Univariate analysis identified a relative tumor volume in the liver $\geq 50 \%(p=0.013)$, AFP $\geq 400 \mathrm{ng} / \mathrm{mL}(p=0.048)$, and SD as the firsttime response of tumor in the MVI $(p=0.002)$ as significant factors predicting unfavorable OS. Extrahepatic spread $(p=0.077)$ showed a tendency for unfavorable OS. 
By multivariate analysis, relative tumor volume in the liver $\geq 50 \%$ (odds ratio [OR], $4.501 ; 95 \%$ confidence interval [CI], 1.61-12.59; $p=0.041$ ) and the first-time response of tumor in the MVI as PD or SD (OR, 7.396; 95\% Cl, 2.639-20.72; $p<0.001)$ remained significant and independent factors for predicting poor OS (Figure $2 \mathrm{~A}$ and $\mathrm{B}$; and Table 3 ). 
Table 3

Risk factors of overall survival in HCC patients with MVI received one-shot CDDP plus RT

\section{Parameter}

Univariate analysis

\section{Multivariate analysis}

OR $\quad 95 \% \mathrm{Cl}$

\section{value}

Age $(\geq 65 /<65$ years $)$

Gender (female/male)

Etiology (with viral hepatitis/without viral hepatitis)
0.303

0.599

\section{ECOG-Performance Status $(0 / \geq 1)$}

Albumin $(\geq 3.5 /<3.5 \mathrm{~g} / \mathrm{dL})$
0.924

0.692

Prothrombin consumption test $(\geq 70 \% /<70 \%)$

0.789

Child-Pugh score $(6 \geq / 5)$

mALBI grade $(\geq 2 b / \leq 2 a)$

0.129

Diameter of main tumor $(\geq 70 /<70 \mathrm{~mm})$

0.11

Number of tumors in the liver $(\geq 4 /<4)$

Relative tumor volume in the liver $(\geq 50 \% /<$ $50 \%)$

$\mathrm{Vp}(3 / 4)$

0.011

$4.501 \quad 1.61-12.59$

0.041

0.801

Vv (with/without)

0.539

Extrahepatic spread (with/without)

Alpha-fetoprotein ( $\geq 400 /<400 \mathrm{ng} / \mathrm{mL}$ )

0.048

0.403

Des-y-carboxy prothrombin $(\geq 400 /<400$ $\mathrm{mAU} / \mathrm{mL}$ )

First response of MVI (SD or PD/ CR or PR)

0.002

7.396

2.63920.72

Univariate and multivariate analysis of risk factor of overall survival in HCC patients with MVI who received one-shot CDDP chemotherapy via HAl plus RT. Abbreviations: HCC, hepatocellular carcinoma; $\mathrm{MVI}$, macrovascular invasion; CDDP, cisplatin; RT, radiation therapy; OR, odds ratio; $\mathrm{Cl}$, confidence interval; ECOG-Performance Status, Eastern Cooperative Oncology Group Performance Status; ALBI score $=\log _{10}([$ total-bilirubin $(\mathrm{mg} / \mathrm{dL})] * 17.1 * 0.66-[$ albumin $(\mathrm{g} / \mathrm{dL})] * 10 * 0.085 ; \mathrm{mALBI}$ grade $1: 2 \mathrm{a}: 2 \mathrm{~b}: 3=$ ALBI score $\leq-2.6$ : $>-2.6$ to $<-2.27$ : $\geq-2.27$ to $\leq-1.39$ : $>-1.39 ; \mathrm{Vp}$, portal vein invasion; $\mathrm{Vv}$, venous invasion; $\mathrm{SD}$, stable disease; $\mathrm{PD}$, progressive disease; $\mathrm{CR}$, complete response; $\mathrm{PR}$, partial response; $\mathrm{HAl}$, hepatic arterial infusion. 


\subsection{PFS and Factors Affecting PFS}

The median PFS time was 3.2 months (Figure 1B). Univariate analysis identified a relative tumor volume in the liver of $\geq 50 \%(p<0.001)$ as a significant predictor of poor PFS. By multivariate analysis, a relative tumor volume in the liver of $\geq 50 \%(\mathrm{OR}, 2.79 ; 95 \% \mathrm{Cl}, 1.247-6.243 ; p=0.013)$ remained a significant and independent factor for predicting unfavorable PFS (Table 4). 
Table 4

Risk factors predicting progression-free survival in HCC patients with MVI received one-shot CDDP plus RT

\begin{tabular}{|c|c|c|c|c|}
\hline \multirow[t]{2}{*}{ Parameter } & \multirow{2}{*}{$\begin{array}{l}\text { Univariate } \\
\text { analysis }\end{array}$} & \multicolumn{3}{|c|}{ Multivariate analysis } \\
\hline & & OR & $95 \% \mathrm{Cl}$ & $\begin{array}{l}\mathrm{p} \\
\text { value }\end{array}$ \\
\hline Age $(\geq 65 /<65$ years $)$ & 0.753 & & & \\
\hline Gender (female/male) & 0.269 & & & \\
\hline $\begin{array}{l}\text { Etiology (with viral hepatitis/without viral } \\
\text { hepatitis) }\end{array}$ & 0.991 & & & \\
\hline ECOG-PS $(0 / \geq 1)$ & 0.799 & & & \\
\hline Albumin $(\geq 3.5 /<3.5 \mathrm{~g} / \mathrm{dL})$ & 0.838 & & & \\
\hline Prothrombin consumption test ( $\geq 70 \% /<70 \%)$ & 0.619 & & & \\
\hline Child-Pugh score $(\geq 6 / 5)$ & 0.375 & & & \\
\hline $\operatorname{mALBI}$ grade $(\geq 2 b / \leq 2 a)$ & 0.505 & & & \\
\hline Diameter of main tumor $(\geq 70 /<70 \mathrm{~mm})$ & 0.479 & & & \\
\hline Number of tumors in the liver $(\geq 4 /<4)$ & 0.201 & & & \\
\hline $\begin{array}{l}\text { Relative tumor volume in the liver }(\geq 50 \% /< \\
50 \%)\end{array}$ & 0.009 & 2.79 & $\begin{array}{l}1.247- \\
6.243\end{array}$ & 0.013 \\
\hline $\operatorname{Vp}(3 / 4)$ & 0.949 & & & \\
\hline Vv (with/without) & 0.713 & & & \\
\hline Extrahepatic spread (with/without) & 0.304 & & & \\
\hline Alpha-fetoprotein ( $\geq 400 /<400 \mathrm{ng} / \mathrm{mL}$ ) & 0.135 & & & \\
\hline $\begin{array}{l}\text { Des-y-carboxy prothrombin }(\geq 400 /<400 \\
\mathrm{mAU} / \mathrm{mL})\end{array}$ & 0.906 & & & \\
\hline Best response of $\mathrm{MVI}$ (CR or PR/SD or PD) & 0.132 & & & \\
\hline \multicolumn{5}{|c|}{$\begin{array}{l}\text { Univariate and multivariate analysis of risk factor of overall survival in HCC patients with MVI who } \\
\text { received one-shot CDDP chemotherapy via HAl plus RT. Abbreviations: HCC, hepatocellular carcinoma, } \\
\text { MVI, macrovascular invasion; CDDP, cisplatin; RT, radiation therapy; OR, odds ratio; Cl, confidence } \\
\text { interval; ECOG-Performance Status, Eastern Cooperative Oncology Group Performance Status; ALBI } \\
\text { score = } \log _{10}\left([\text { total-bilirubin }(\mathrm{mg} / \mathrm{dL})]^{\star} 17.1^{*} 0.66 \text { - } \text {-albumin }(\mathrm{g} / \mathrm{dL})\right]^{*} 10^{*} 0.085 ; \mathrm{mALBI} \text { grade } 1: 2 \mathrm{a}: 2 \mathrm{~b}: 3=\end{array}$} \\
\hline
\end{tabular}




\subsection{Treatment-Related Adverse Events}

Treatment-related toxicities were observed in 16 of 32 patients (50\%). The common adverse events were fever ( $25 \%, 8$ cases), elevated transaminase level (16\%, 5 cases), anorexia (6\%, 2 cases), elevated ammonia level $(6 \%, 2$ cases), ascites $(3 \%, 1$ case), and general fatigue (3\%,1 case). The adverse events in this study were categorized as CTCAE grades 1 or 2 . None of the patients who received chemotherapy via HAI and RT for advanced HCC with MVI developed hepatic failure that met the criteria for classical or nonclassical RILD (Table 5).

Table 5

Adverse events after administration of one-shot CDDP via HAl and RT, as categorized by NCI-CTCAE

\begin{tabular}{|lll|}
\hline & All (N=32) & \\
\hline Adverse event & Any grade & Grade $\geq 3$ \\
\hline Fever & $25(8)$ & 0 \\
\hline Aspartate aminotransferase increased & $16(5)$ & 0 \\
\hline Alanine aminotransferase increased & $12(4)$ & 0 \\
\hline Anorexia & $6(2)$ & 0 \\
\hline Elevated ammonia & $6(2)$ & 0 \\
\hline Ascites & $3(1)$ & 0 \\
\hline Fatigue & $3(1)$ & 0 \\
\hline $\begin{array}{l}\text { The adverse events after administration of one-shot CDDP via HAl and RT, as categorized by NCl- } \\
\text { CTCAE. Data are presented as percentages (numbers). Abbreviations: CDDP, cisplatin; HAl, hepatic } \\
\text { arterial infusion; RT, radiation therapy; NCl-CTCAE, National Cancer Institute Common Terminology } \\
\text { Criteria for Adverse Event. }\end{array}$ \\
\hline
\end{tabular}

\section{Discussion}

In a previous study, we reported that the combination of HAl via the reservoir system and RT for patients with HCC plus MVI led to improved outcomes [26-28]. In this study, we analyzed the clinical outcomes of patients with HCC with MVI treated by the combination of one-shot CDDP administered via HAI plus RT. The median OS and PFS times were 8.6 and 3.2 months, respectively. The ORR, evaluated 1.4 months after HAl, was $16 \%$ for the main tumor, and $59 \%$ for tumor in the MVI. There were no severe adverse events that ended the study for any of the patients. Nineteen of 32 patients received additional systemic therapy. These results seemed well tolerated and showed immediate and favorable control of MVI. Since 
we did not use the implanted reservoir system in this study, there were no adverse events associated with an implanted port and catheter which we reported in previous study with reservoir system.

HCC is a leading cause of cancer-related death worldwide, and the prognosis of patients with unresectable HCC with major MVI is extremely poor $[1,2]$. Systemic chemotherapy, such as atezo+beva, is effective for advanced HCC $[10,23]$ and for HCC with Vp. However, the prognosis remains poor, with $67 \%$ of patients alive 6 months after the initiation of treatment with atezo+beva [24,25]. Kudo et al. reported that MVI is a factor leading to poor outcomes in patients with advanced HCC because MVI rapidly worsens flow in the portal vein and leads to liver failure and portal hypertension [10].

Many studies have reported the efficacy of HAl for treating patients with HCC with the portal vein tumor thrombus (PVTT). This HAl regimen was based on 5-fuluolouracil (5-FU) and cisplatin (FP), and infused repeatedly via an implanted catheter and port which called reservoir system. The prospective SILIUS study, which compared sorafenib and HAI with low-dose FP did not show better results than those of the patients treated with sorafenib; however, the subgroup analysis of patients with HCC with Vp4 showed a better outcome than that of patients treated with sorafenib [28]. Fujino et al. reported that the combination of HAl with 5-FU based regimen and RT showed better ORR for patients with HCC with PVTT than with HAI without RT [26]. Kodama et al. compared the combination of HAI and RT for patients with HCC with PVTT and sorafenib without RT and found respective median OS times of 9.9 vs. 5.3 months [29]. Kawaoka et al. compared the outcomes of the patients treated with 5-FU regimen administered by $\mathrm{HAl}$ and one-shot CDDP administered by HAl, and showed a comparison of the ORR and median OS time (9.1 vs. 8.6 months), but there was no statically significant difference [30]. Kosaka et al. reported that the OS and PFS of 5-FU via HAl and RT for patients with HCC with Vp4 were 12.1 and 4.2 months, respectively, with 19.6\% ORR for the main tumor, and 51\% ORR for tumor in the MVI [31]. However, there are some problems related to the implanted reservoir system [26,29-31]. Each type of HAI and RT therapy showed a rapid effect for $\mathrm{MVI}$; on the other hand, there was insufficient overall control of the HCC, especially with large intrahepatic volumes over half of the liver or extrahepatic metastasis. Thus, we should consider the rapid initiation of systemic chemotherapy in patients with such advanced HCC.

The current first choice of systemic chemotherapy is atezo+beva $[10,23]$. The median OS of this combination therapy was 19.2 months, which was longer than the median OS of 13.6 months for the sorafenib group [23]. However, the subgroup analysis reported that the prognosis of the HCC patients with $\mathrm{Vp} 4$ remains poor, the median OS of the Vp4 patients treated with atezo+beva was7.6 months and that with sorafenib was 5.5 months [24]. In addition, the median time of response to atezo+beva was 2.8 months after initiation, and approximately $20 \%$ of patients were nonresponders [25]. These timelapse of atezo+beva might increase the risk to improve the MVI and reduce the liver preservation function.

Above all, we suggest that the combination of $\mathrm{HAl}$ and RT is important for the rapid control of MVI and as a bridging therapy to systemic chemotherapy. In particular, one-shot CDDP via HAI combined with RT should be a good therapeutic option because problems related to the reservoir system cannot occur. 
This study has limitations. It was a retrospective single-arm study with a small number of patients. Accumulation of sample cases and further studies such as prospective, multi-arm studies, in addition to systemic reviews, should be considered. We hope that the results of our study warrant further studies.

\section{Conclusion}

The combination of one-shot CDDP chemotherapy via HAI plus RT for patients with HCC and MVI showed rapid control of the MVI. No severe adverse events were observed in this study. Further studies should help to address the poor outcomes of HCC patients with MVI progression who only receive systemic chemotherapy.

\section{Abbreviations}

HCC, hepatocellular carcinoma; MVI, macrovascular invasión; CDDP, cisplatin; HAl, hepatic arterial infusion; RT, radiation therapy; atezo+beva, atezolizumab plus bevacizumab; Vp, portal vein invasion; OS, overall survival; Vp3, tumor thrombus in the hemilobar branch of the portal vein; $\mathrm{Vp} 4$, tumor thrombus in the main trunk or bilobar branches of the portal vein; Vv2,tumor thrombus in the main trunk of left hepatic vein, middle hepatic vein, or right hepatic vein; $V v 3$, tumor thrombus in inferior vena cava; one-shot CDDP, single bolus injection of CDDP; CT, computed tomography; 3D-CRT, three-dimentional conformal radiation therapy; MV, Mega Volt; $\mathrm{mRECIST}$, modified Response Evaluation Criteria in Solid Tumors; $\mathrm{NCl}$ CTCAE, National Cancer Institute Common Terminology Criteria for Adverse Event; RILD, radiation-induced liver damage; PFS, progression-free survival; PD, progressive disease; AFP, alpha-fetoprotein; ECOGPerformance Status, Eastern Cooperative Oncology Group Performance Status; HBV, hepatitis B virus; $\mathrm{HCV}$, hepatitis $C$ virus; $A L B I$ score $=\log _{10}([$ total-bilirubin $(\mathrm{mg} / \mathrm{dL})] * 17.1 * 0.66$-[albumin $(\mathrm{g} / \mathrm{dL})] * 10 * 0.085$; mALBI grade 1: 2a: $2 \mathrm{~b}: 3$ = ALBI score $\leq-2.6$ : $>-2.6$ to <-2.27: $\geq-2.27$ to $\leq-1.39$ : $>-1.39 ; \mathrm{Vv}$, venous invasion; BCLC stage, Barcelona Clinic liver cancer stage; ORR, overall response rates; DCR, disease control rates; $\mathrm{CR}$, complee response; $\mathrm{PR}$, partial response; SD, stable disease; OR, odds ratio; $\mathrm{Cl}$, confidence interval; PVTT, portal vein tumor thrombus; 5-FU, 5-fluoluracil; FP, 5-fluolouracil and cisplatin;

\section{Declarations}

\section{Ethics approval and consent to participate:}

This study was approved by the Institutional Review Board of Hiroshima University Hospital, and was based on the ethical principles of the Declaration of Helsinki $7^{\text {th }}$ version on 2013 (project identification code number: E-968). Detailed written informed consent was obtained from each patient for each treatment. 


\section{Consent for publication:}

Not applicable.

\section{Availability of data and materials:}

The datasets generated and analyzed during the current study are not publicly available due to lack of informed consent for public reuse of datasets but are available from the corresponding author on reasonable request for further studies.

\section{Competing Interest:}

Michio Imamura has received honoraria from Bristol-Myers Squibb and research funding from BristolMyers Squibb. Kazuaki Chayama has received honoraria from MSD K.K., Bristol-Myers Squibb, Gilead Sciences and AbbVie; and research funding from Dainippon Sumitomo Pharma, TORAY, Eisai, Otsuka Pharma, Mitsubishi Tanabe Pharma, Daiichi Sankyo and Bristol-Myers Squibb; and has received research funding from Janssen, Mitsubishi Tanabe, Dainippon Sumitomo, and TORAY. Hiroshi Aikata has received honoraria from Eisai, and Bayer.

\section{Funding:}

This research did not receive external funding.

\section{Author Contributions:}

Study design, data analysis and writing, K.N. and T.K, data acquisition, K.N, statistical analysis, K.N; results review, T.K. and H.A, manuscript revision for important intellectual, H.A.

\section{Acknowledgments:}

Not applicable.

\section{References}

1. Jemal A, Bray F, Center MM, Ferlay J, Ward E, Forman D. Global Cancer Statistics. C.A. Cancer J. Clin. 2011, 61, 69-90.

2. Kamangar F, Dores G.M, Anderson W.F. Patterns of Cancer Incidence, Mortality, and Prevalence Across Five Continents: Defining Priorities to Reduce Cancer Disparities in Different Geographic Regions of the World. J. Clin. Oncol. 2006, 24, 2137-2150. 
3. Okita K. Management of Hepatocellular Carcinoma in Japan. J. Gastroenterol. 2006, 41, 100-106.

4. Hasegawa K, Kokudo N, Makuuchi M, Izumi N, Ichida T, Kudo M, et al. Comparison of Resection and Ablation for Hepatocellular Carcinoma: a Cohort Study Based on a Japanese Nationwide Survey. J. Hepatol. 2013, 58, 724-729.

5. Rossi S, Di Stasi M, Buscarini E, Quaretti P, Garbagnati F, Squassante L, et al. Percutaneous RF Interstitial Thermal Ablation in the Treatment of Hepatic Cancer. A.J.R. Am. J. Roentgenol. 1996, 167, 759-768.

6. Llovet JM, Real MI, Montaña X, Planas R, Coll S, Aponte J, Barcelona Liver Cancer Group, et al. Arterial Embolisation or Chemoembolisation Versus Symptomatic Treatment in Patients with Unresectable Hepatocellular Carcinoma: a Randomised Controlled Trial. Lancet 2002, 359, 17341739.

7. Takayasu K, Arii S, Kudo M, Ichida T, Matsui O, Izumi N, et al. Superselective Transarterial Chemoembolization for Hepatocellular Carcinoma. Validation of Treatment Algorithm Proposed by Japanese Guidelines. J. Hepatol. 2012, 56, 886-892.

8. Kim DY, Park W, Lim DH, Lee JH, Yoo BC, Paik SW, et al. Three-Dimensional Conformal Radiotherapy for Portal Vein Thrombosis of Hepatocellular Carcinoma. Cancer 2005, 103, 2419-2426.

9. Ando E, Yamashita F, Tanaka M, Tanikawa K. A Novel Chemotherapy for Advanced Hepatocellular Carcinoma with Tumor thrombusof the Main Trunk of the Portal Vein. Cancer 1997, 79, 1890-1896.

10. Kudo M. A Paradigm Change in the Treatment Strategy for Hepatocellular Carcinoma. Liver Cancer 2020, 9, 367-377.

11. Cheng AL, Kang YK, Chen Z, Tsao CJ, Qin S, Kim JS, et al. Efficacy and Safety of Sorafenib in Patients in the Asia-Pacific Region with Advanced Hepatocellular Carcinoma: a phase III Randomized, Double-Blind, Placebo-Controlled Trial. Lancet Oncol. 2009, 10, 25-34.

12. Abou-Alfa GK, Amadori D, Santoro A, Figer A, De Greve J, Lathia C, et al. Safety and Efficacy of Sorafenib in Patients with Hepatocellular Carcinoma (HCC) and Child-Pugh A Versus B Cirrhosis. Gastrointest. Cancer Res. 2011, 4, 40-44.

13. Hollebecque A, Cattan S, Romano O, Sergent G, Mourad A, Louvet A, et al. Safety and Efficacy of Sorafenib in Hepatocellular Carcinoma: the Impact of the Child-Pugh Score. Aliment. Pharmacol. Ther. 2011, 34, 1193-1201.

14. Itamoto T, Nakahara H, Tashiro H, Haruta N, Asahara T, Naito A, et al. Hepatic Arterial Infusion of 5Fluorouracil and Cisplatin for Unresectable or Recurrent Hepatocellular Carcinoma with Tumor Thrombus of the Portal Vein. J. Surg. Oncol. 2002, 80, 143-148.

15. Sakon M, Nagano H, Dono K, Nakamori S, Umeshita K, Yamada A, et al. Combined Intraarterial 5Fluorouracil and Subcutaneous Interferon-Alpha Therapy for Advanced Hepatocellular Carcinoma with Tumor Thrombi in the Major Portal Branches. Cancer 2002, 94, 435-442.

16. Obi S, Yoshida H, Toune R, Unuma T, Kanda M, Sato S, et al. Combination Therapy of Intraarterial 5Fluorouracil and Systemic Interferon-Alpha for Advanced Hepatocellular Carcinoma with Portal Venous Invasion. Cancer 2006, 106, 1990-1997. 
17. Ota $H$, Nagano $H$, Sakon M, Eguchi $H$, Kondo $M$, Yamamoto $T$, et al. Treatment of Hepatocellular Carcinoma with Major Portal Vein Thrombosis by Combined Therapy with Subcutaneous InterferonAlpha and Intra-Arterial 5-Fluorouracil; Role of type 1 Interferon Receptor Expression. Br. J. Cancer 2005, 93, 557-564.

18. Uka K, Aikata H, Takaki S, Miki D, Jeong SC, Hiramatsu A, et al. Similar Side Effects of Recombinant Interferon-alpha-2b and Natural Interferonalpha When Combined with Intra-Arterial 5-Fluorouracil for the Treatment of Advanced Hepatocellular Carcinoma. Liver Int. 2007, 27, 1209-1216.

19. Toya R, Murakami R, Baba Y, Nishimura R, Morishita S, lkeda O, et al. Conformal Radiation Therapy for Portal Vein Tumor thrombusof Hepatocellular Carcinoma. Radiother. Oncol. 2007, 84, 266-271.

20. Han KH, Seong J, Kim JK, Ahn SH, Lee DY, Chon CY. Pilot Clinical Trial of Localized Concurrent Chemoradiation Therapy for Locally Advanced Hepatocellular Carcinoma with Portal Vein Thrombosis. Cancer 2008, 113, 995-1003.

21. Katamura Y, Aikata H, Takaki S, Azakami T, Kawaoka T, Waki K, et al. Intra-Arterial 5Fluorouracil/Interferon Combination Therapy for Advanced Hepatocellular Carcinoma with or Without Three-dimensional Conformal Radiotherapy for Portal Vein Tumor Thrombosis. J. Gastroenterol. 2009, 44, 492-502.

22. Liver Cancer Study Group of Japan. Guidelines for EvidenceBased Clinical Practice for the Treatment of Liver Cancer. Clinical Practice Guidelines for Hepatocellular Carcinoma; Kanehara \& Co., Ltd.: Tokyo, 2013. (in Japanese).

23. Finn RS, Qin S, Ikeda M, Galle PR, Ducreux M, Kim TY, et al. Atezolizumab plus Bevacizumab in Unresectable Hepatocellular Carcinoma. N. Engl. J. Med. 2020, 382, 1894-1905.

24. Finn RS, Qin S, Ikeda M, Galle PR, Ducreux M, Kim TY, et al. IMbrave150: Updated Overall Survival (OS) Data from a Global, Randomized, Open-Label phase III Study of Atezolizumab (Atezo) + Bevacizumab (Bev) Versus Sorafenib (Sor) in Patients (pts) with Unresectable Hepatocellular Carcinoma (HCC). J. Clin. Oncol. 2021, 39, 267-267.

25. Breder V, Arndt V, Philippe M, et al. IMbrave150: Exploratory efficacy and safety results of hepatocellular carcinoma (HCC) patients (pts) with main trunk and/or contralateral portal vein invasion (Vp4) treated with atezolizumab (atezo) + bevacizumab (bev) versus sorafenib (sor) in a global Ph III study. J. Clin. Oncol. 2021, 39, 4073-4073

26. Fujino H, Kimura T, Aikata H, Miyaki D, Kawaoka T, Kan H, et al. Role of 3-D Conformal Radiotherapy for Major Portal Vein Tumor thrombusCombined with Hepatic Arterial Infusion Chemotherapy for Advanced Hepatocellular Carcinoma. Hepatol. Res. 2015, 45, 607-617.

27. Ikeda M, Shimizu S, Sato T, Morimoto M, Kojima Y, Inaba Y, et al. Sorafenib plus Hepatic Arterial Infusion Chemotherapy with Cisplatin Versus Sorafenib for Advanced Hepatocellular Carcinoma: Randomized phase II Trial. Ann. Oncol. 2016, 27, 2090-2096.

28. Kudo M, Ueshima K, Yokosuka O, Ogasawara S, Obi S, Izumi N, et al. Sorafenib plus Low-Dose Cisplatin and Fluorouracil Hepatic Arterial Infusion Chemotherapy Versus Sorafenib Alone in Patients 
with Advanced Hepatocellular Carcinoma (SILIUS): a Randomised, Open Label, phase 3 Trial. Lancet Gastroenterol. Hepatol. 2018, 3, 424-432.

29. Kodama K, Kawaoka T, Aikata H, Uchikawa S, Nishida Y, Inagaki Y, et al. Comparison of Outcome of Hepatic Arterial Infusion Chemotherapy Combined with Radiotherapy and Sorafenib for Advanced Hepatocellular Carcinoma Patients with Major Portal Vein Tumor Thrombosis. Oncology 2018, 94, 215-222.

30. Kawaoka T, Aikata H, Kobayashi T, Uchikawa S, Ohya K, Kodama K, et al. Comparison of Hepatic Arterial Infusion Chemotherapy Between 5-Fluorouracil-Based Continuous Infusion Chemotherapy and Low-Dose Cisplatin Monotherapy for Advanced Hepatocellular Carcinoma. Hepatol. Res. 2018, $48,1118-1130$.

31. Kosaka Y, Kimura T, Kawaoka T, Ogawa Y, Amioka K, Naruto K, et al. Hepatic Arterial Infusion Chemotherapy Combined with Radiation Therapy for Advanced Hepatocellular Carcinoma with Tumor thrombusof the Main Trunk or Bilobar of the Portal Vein. Liver Cancer 2021, 10, 151-160.

32. Eisenhauer EA, Therasse P, Bogaerts J, Schwartz LH, Sargent D, Ford R, et al. New response evaluation criteria in solid tumors: revised RECIST guideline, 1.1 version. Eur J Cancer 2009, 45, 228247.

33. Pan CC, Kavanagh BD, Dawson LA, Li XA, Das SK, Miften M, et al. Radiation associated Liver Injury. Int. J. Radiat. Oncol. Biol. Phys. 2010, 76, S94-S100.

34. Kanda Y. Investigation of the Freely Available Easy-to-Use Software "EZR" (Easy R) for Medical Statistics. Bone Marrow Transplant. 2013, 48, 452-458.

\section{Figures}


(A)

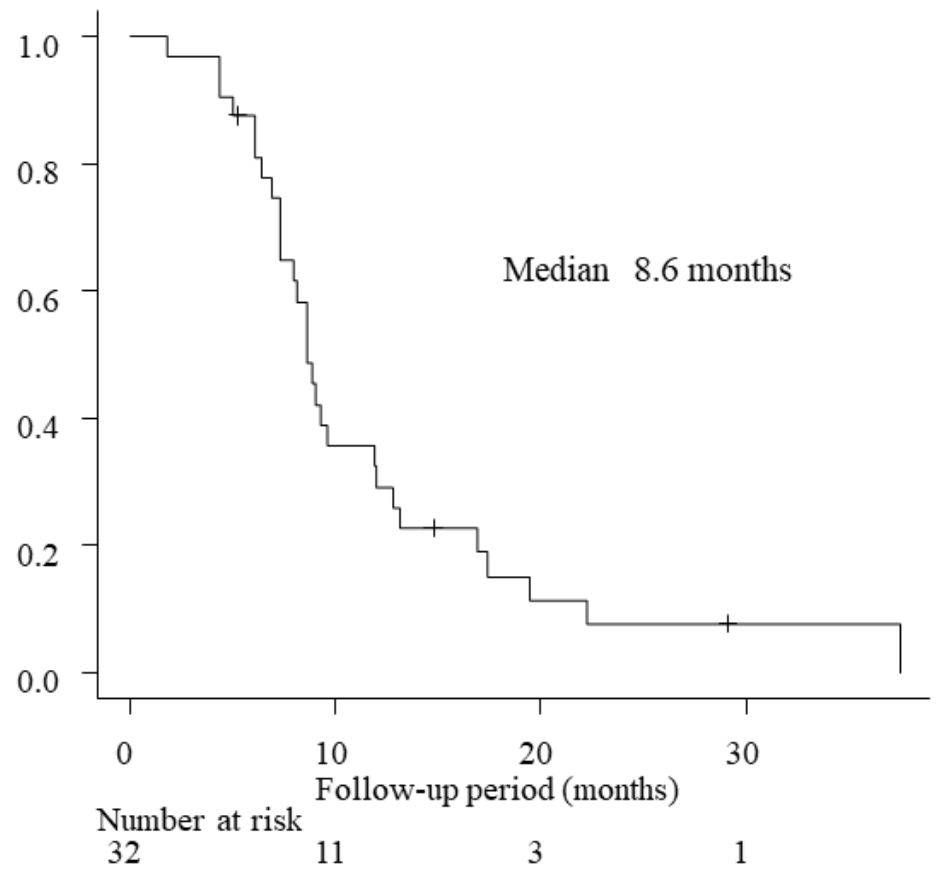

(B)

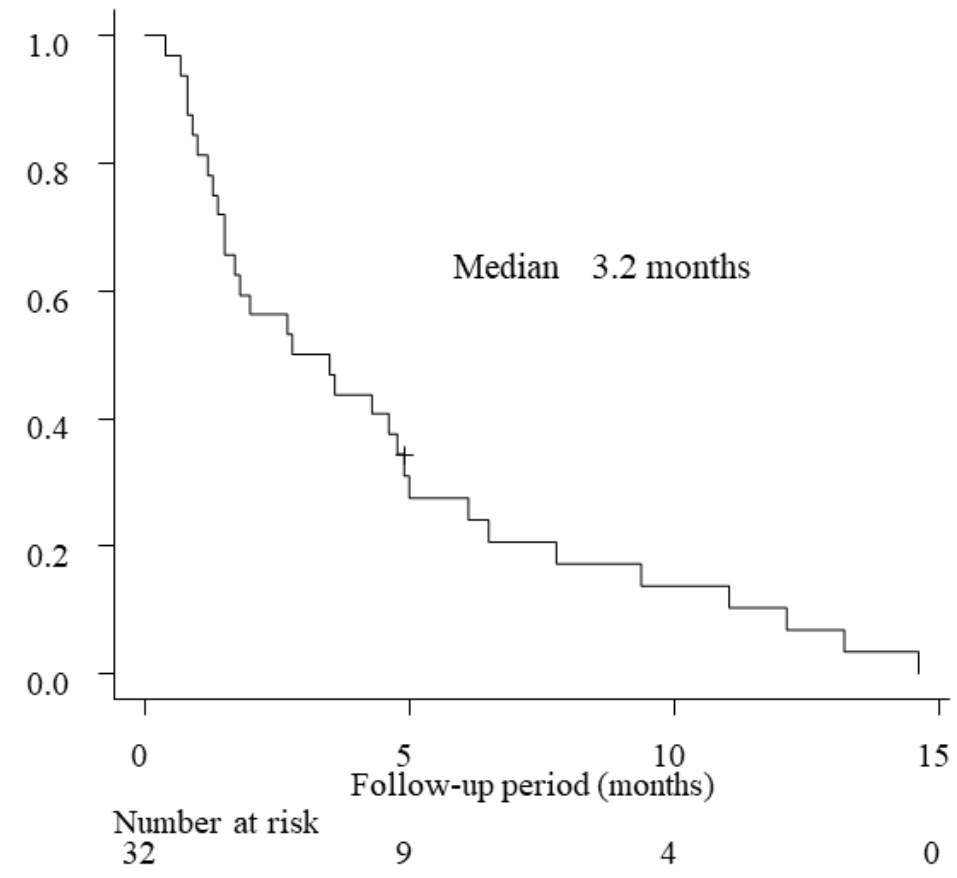

\section{Figure 1}

(A) Overall survival of patients with HCC treated with the combination of one-shot CDDP via HAI and RT.

(B) Progression-free survival of patients with HCC treated with the combination of one-shot CDDP via HAI and RT. Abbreviations: HCC, hepatic cell carcinoma; CDDP, cisplatin; HAl, hepatic arterial infusion; RT, radiation therapy.

(A)

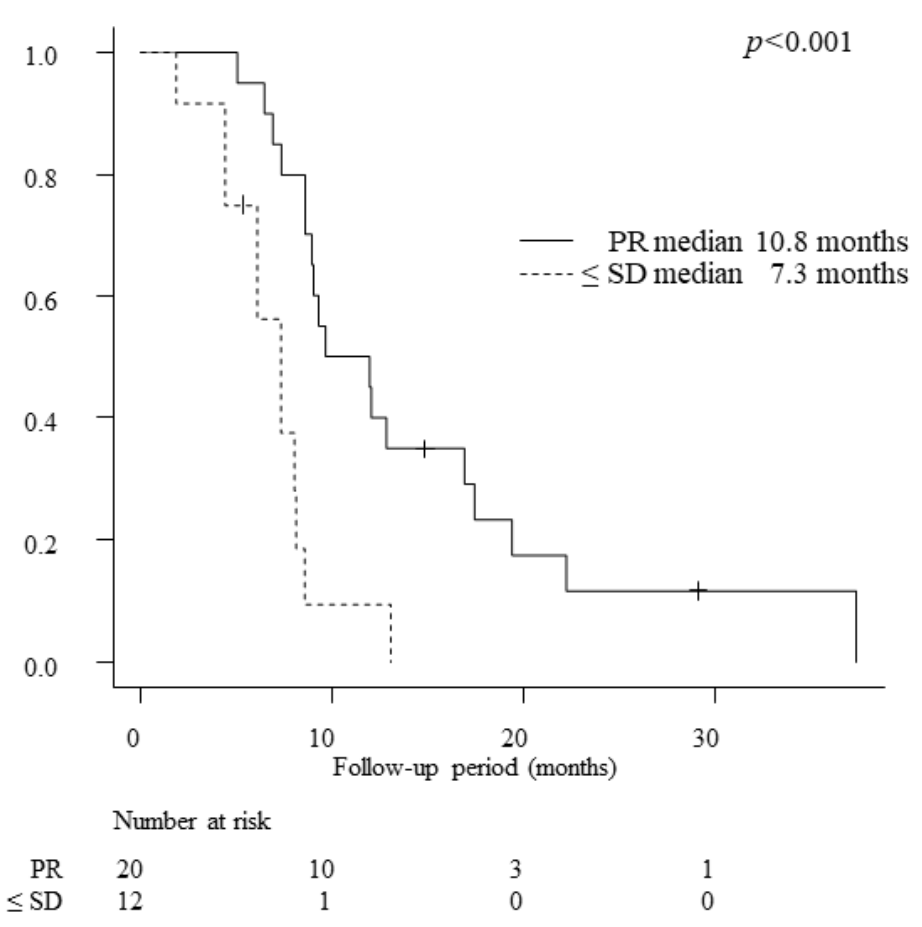

(B)

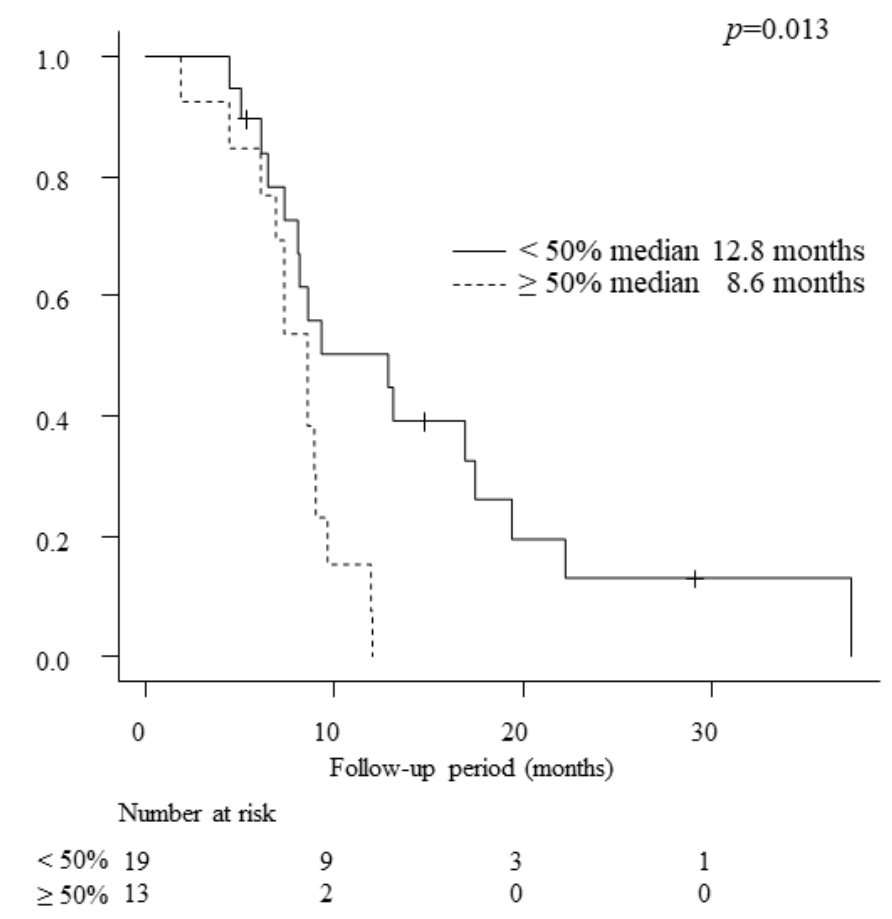


Figure 2

(A) Overall survival of patients with HCC treated with the combination of one-shot CDDP chemotherapy via $\mathrm{HAl}$ and RT, divided by the first-time response of tumor thrombus in the portal vein PR/SD. (B) Overall survival of patients with HCC treated with the combination of one-shot CDDP chemotherapy via HAI and $\mathrm{RT}$, divided by the relative tumor volume $<50 \% / \geq 50 \%$. Abbreviations: $\mathrm{HCC}$, hepatocellular carcinoma; CDDP, cisplatin; HAl, hepatic arterial infusion; RT, radiation therapy; PR, partial response; SD, stable disease. 